\title{
GLOBAL ATTRACTIVITY IN NONLINEAR DELAY DIFFERENCE EQUATIONS
}

\author{
V. LJ. KOCIĆ AND G. LADAS \\ (Communicated by Charles Pugh)
}

\begin{abstract}
We obtain a set of sufficient conditions under which all positive solutions of the nonlinear delay difference equation $x_{n+1}=x_{n} f\left(x_{n-k}\right), n=$ $0,1,2, \ldots$, are attracted to the positive equilibrium of the equation. Our result applies, for example, to the delay logistic model $N_{t+1}=\alpha N_{t} /\left(1+\beta N_{t-k}\right)$ and to the delay difference equation $x_{n+1}=x_{n} e^{r\left(1-x_{n-k}\right)}$.
\end{abstract}

\section{INTRODUCTION AND PRELIMINARIES}

Our aim in this paper is to establish the following global attractivity result for the nonlinear delay difference equation

$$
x_{n+1}=x_{n} f\left(x_{n-k}\right), \quad n=0,1,2, \ldots
$$

Theorem 1. Assume that $k$ is a positive integer and that $f$ satisfies the following properties:

(2)(i) $\quad f \in \mathbf{C}[[0, \infty),(0, \infty)]$ and $f(u)$ is nonincreasing in $u$;

(ii) The equation $f(x)=1$ has a unique positive solution;

(iii) If $\bar{x}$ denotes the unique positive solution of $f(x)=1$ then

$$
[x f(x)-\bar{x}](x-\bar{x})>0 \quad \text { for } x \neq \bar{x}
$$

(iv) The only solution of the equation

$$
\bar{x}\left[f\left(\bar{x}(f(x))^{k}\right)\right]^{k}=x
$$

in the interval $0 \leq x \leq \bar{x}(f(0))^{k}$ is $x=\bar{x}$.

Then $\bar{x}$ is a global attractor of all positive solutions of (1).

In $\S 3$ we show that when $k=1$, condition (iv) of Theorem 1 is a direct consequence of conditions (i)-(iii). It will also be clear from the proof of Theorem 1 that the conclusion of the theorem remains true if conditions (iii) and (iv) are replaced by the following condition:

Received by the editors February 5, 1991.

1991 Mathematics Subject Classification. Primary 39A12.

Key words and phrases. Global attractivity, higher order nonlinear difference equation.

The first author is on leave from the Department of Mathematics, Faculty of Electrical Engineering, University of Belgrade, 11000 Belgrade, Yugoslavia. 
(iii') The only solution of the equation $\bar{x}\left[f\left(\bar{x}\left(f(x)^{k+1}\right)\right]^{k+1}=x\right.$ in the interval $0 \leq x \leq \bar{x}(f(0))^{k+1}$ is $x=\bar{x}$.

By a solution of (1) we mean a sequence $\left\{x_{n}\right\}$ that is defined for $n \geq-k$ and that satisfies (1) for $n \geq 0$. If $a_{-k}, a_{-k+1}, \ldots, a_{0}$ are $(k+1)$ given nonnegative numbers, then (1) has a unique solution satisfying the initial conditions

$$
x_{i}=a_{i} \quad \text { for } i=-k, \ldots, 0 \text {. }
$$

If

$$
a_{-k}, \ldots, a_{-1} \in[0, \infty) \text { and } a_{0} \in(0, \infty)
$$

then, clearly, the solution of the initial value problem (1) and (5) is positive for $n \geq 0$. In this paper, we will only investigate solutions of (1) whose initial values satisfy condition (6). Such solutions will also be called positive solutions.

An immediate application of Theorem 1 is to the discrete delay logistic model

$$
N_{t+1}=\frac{\alpha N_{t}}{1+\beta N_{t-k}},
$$

which was proposed by Pielou in her books [6, p. 22; 7, p. 79] as a discrete analog of the delay logistic equation

$$
\dot{N}(t)=r N(t)\left[1-\frac{N(t-\tau)}{P}\right] \text {. }
$$

Theorem 1 can also be applied to the more general discrete delay model

$$
N_{n+1}=\frac{\alpha N_{n}}{\left(1+\alpha N_{n-k}\right)^{b}+\beta N_{n-k}}, \quad n=0,1,2, \ldots,
$$

which for $k=0$ is the population model for annual plants that was derived in [8].

For $k=1$, Theorem 1 was established by a different proof in [3]. The local stability analysis for biological models described by (1) was presented by Levin and May in [5]. See also Bergh and Getz [1].

Some powerful and elegant global stability results for difference equations were recently obtained by Karakostas, Philos, and Sficas [2]; however, their range of applicability is different from the results in this paper.

We hope that our results will be useful in investigating the global stability of difference equations in which, according to Levin and May [5], the density dependent mechanisms themselves operate with an explicit time delay of $T$ generations; that is,

$$
N_{t+1}=N_{t} F\left(N_{t-T}\right)
$$

\section{Proof OF THEOREM 1}

Let $\left\{x_{n}\right\}$ be a positive solution of (1). We must prove that

$$
\lim _{n \rightarrow \infty} x_{n}=\bar{x} \text {. }
$$

There are only two cases to consider.

Case 1. For $n$ sufficiently large,

$$
\begin{aligned}
& \text { either } x_{n} \geq \bar{x} \\
& \text { or } x_{n} \leq \bar{x} .
\end{aligned}
$$


We will assume that (10) holds. The case where (11) holds is similar and is omitted. Then it follows from (1) that for $n$ sufficiently large, $\left\{x_{n}\right\}$ is eventually decreasing. Let $b=\lim _{n \rightarrow \infty} x_{n}$. Then $b>0$ and by taking limits on both sides of (1) we find that $f(b)=1$. This implies that $b=\bar{x}$, which completes the proof in this case.

Case 2. The sequence $\left\{x_{n}\right\}$ is "strictly" oscillatory about the positive equilibrium $\bar{x}$ in the following sense: There exists a sequence of positive integers $\left\{n_{i}\right\}$ for $i=1,2, \ldots$ such that $k<n_{1}<n_{2}<\cdots, \lim _{i \rightarrow \infty} n_{i}=\infty, x_{n_{i}}<\bar{x}$ and $x_{n_{i}+1} \geq \bar{x}$ for $i=1,2, \ldots$, and for each $i=1,2, \ldots$ some of the terms $x_{j}$ with $n_{i}<j \leq n_{i+1}$ are greater and some are less than $\bar{x}$.

For each $i=1,2, \ldots$ let $m_{i}$ and $M_{i}$ be any. integers in the interval $\left(n_{i}, n_{i+1}\right)$ such that

$$
x_{m_{i}}=\min \left\{x_{j} \text { for } n_{i}<j \leq n_{i+1}\right\}
$$

and

$$
x_{M_{i}}=\max \left\{x_{j} \text { for } n_{i}<j \leq n_{i+1}\right\} .
$$

Then for each $i=1,2, \ldots$

$$
x_{m_{i}}<\bar{x}, \quad x_{m_{i}} \leq x_{m_{i}-1}
$$

and

$$
x_{M_{i}}>\bar{x}, \quad x_{M_{i}} \geq x_{M_{i}-1} .
$$

Now we claim that the following statements are true for each $i=1,2, \ldots$ :

(i) There exists $p_{i} \in \mathbb{N}$ with $\max \left\{n_{i}, M_{i}-(k+1)\right\} \leq p_{i}<M_{i}$ such that

$$
x_{p_{i}} \leq \bar{x} \text { and } x_{j}>\bar{x} \text { for } j=p_{i}+1, \ldots, M_{i} \text {. }
$$

(ii) There exists $q_{i} \in \mathbb{N}$ with $\max \left\{n_{i}, m_{i}-(k+1)\right\} \leq q_{i}<m_{i}$ such that

$$
x_{q_{i}} \geq \bar{x} \text { and } x_{j}<\bar{x} \text { for } j=q_{i}+1, \ldots, m_{i} \text {. }
$$

We will only prove (i). The proof of (ii) is similar and is omitted. To this end, it suffices to show that there exists at least one $\bar{p}_{i} \in \mathbb{N}$ such that $x_{\bar{p}_{i}} \leq \bar{x}$ with $\max \left\{n_{i}, M_{i}-(k+1)\right\} \leq \bar{p}_{i}<M_{i}$. Then the desired $p_{i}$ of statement (i) will be the greatest of all such $\bar{p}_{i}$ 's. Assume, for the sake of contradiction, that there exists no such $\bar{p}_{i}$. Then $n_{i}<M_{i}-(k+1)$ and $x_{j}>\bar{x}$ for all $j$ in the interval $\left[M_{i}-(k+1), M_{i}\right]$. Furthermore, since $x_{M_{i}-(k+1)}>\bar{x}$ and $f$ is nonincreasing, it follows that $f\left(x_{M_{i}-(k+1)}\right) \leq f(\bar{x})=1$. Clearly, the above inequality must be strict, for otherwise, $f(x)=1$ would have more than one positive solution. Hence

$$
X_{M_{i}-1} \leq x_{M_{i}}=x_{M_{i}-1} f\left(x_{M_{i}-(k+1)}\right)<x_{M_{i}-1} \text {. }
$$

This is a contradiction and the proof of (i) is complete.

Let

$$
\mu=\limsup _{n \rightarrow \infty} x_{n}=\limsup _{i \rightarrow \infty} x_{M_{i}}
$$

and

$$
\lambda=\liminf _{n \rightarrow \infty} x_{n}=\lim _{i \rightarrow \infty} \inf x_{m_{i}}
$$


To prove that (9) holds, it suffices to show that $\lambda=\mu=\bar{x}$. First assume that $\lambda>0$. Then from (12) and (13) it follows that given $\eta<0$ and $\varepsilon>0$, with $0<\varepsilon<\lambda$, there exists $n_{0} \in \mathbb{N}$ such that

$$
\lambda-\varepsilon \leq x_{n} \leq \mu+\eta \text { for } n \geq n_{0}-k .
$$

By multiplying the equations that result from (1) from $n=p_{i}$ to $n=M_{i}-1$, for $i$ sufficiently large, we obtain

$$
x_{M_{i}}=x_{p_{i}} \Pi_{j=p_{i}}^{M_{i}-1} f\left(x_{j-k}\right) .
$$

Now recall that $0<M_{i}-p_{i} \leq k+1$. If $M_{i}-p_{i}<k+1$, then since $x_{p_{i}} \leq \bar{x}$ and $x_{j-k} \geq \lambda-\varepsilon$ for $j=p_{i}+1, \ldots, M_{i}-1,(14)$ yields

$$
x_{M_{i}} \leq \bar{x}(f(\lambda-\varepsilon))^{k} .
$$

On the other hand, if $M_{i}-p_{i}=k+1$ then (14) can be rewritten as

$$
x_{M_{i}}=x_{p_{i}} f\left(x_{p_{i}}\right) \sqcap_{j=p_{i}}^{M_{i}-2} f\left(x_{j-k}\right),
$$

and so (15) also holds because, by (3), $x_{p_{i}} f\left(x_{p_{i}}\right) \leq \bar{x}$ and

$$
f\left(x_{j-k}\right) \leq f(\lambda-\varepsilon) \quad \text { for } j=p_{i}+1, \ldots, M_{i}-2 .
$$

As (15) holds for every $\varepsilon \in(0, \lambda)$ we conclude that

$$
\mu \leq \bar{x}[f(\lambda)]^{k} .
$$

If $\lambda=0$ one can see that (15) is also true with $\lambda-\varepsilon$ replaced by 0 . Therefore (16) is true for any $\lambda \geq 0$.

In a similar way, by multiplying the equations that result from (1) from $n=q_{i}$ to $n=m_{i}-1$, for $i$ sufficiently large, and then by simplifying as above, we are led to the estimate

$$
\lambda \geq \bar{x}[f(\mu)]^{k} .
$$

Set $L_{1}=0, R_{1}=\bar{x}[f(0)]^{k}$, and for $m=1,2, \ldots$,

$$
L_{m+1}=\bar{x}\left[f\left(R_{m}\right)\right]^{k} \quad \text { and } \quad R_{m}=\bar{x}\left[f\left(L_{m}\right)\right]^{k} .
$$

Then one can show by induction that $\left\{R_{m}\right\}$ is a decreasing sequence, $\left\{L_{m}\right\}$ is an increasing sequence, and for $m=1,2, \ldots, L_{m} \leq \lambda \leq \mu \leq R_{m}$.

Set $L=\lim _{m \rightarrow \infty} L_{m}$ and $R=\lim _{m \rightarrow \infty} R_{m}$. Then, by taking limits, on both sides of (18) we find

$$
L=\bar{x}[f(R)]^{k} \quad \text { and } \quad R=\bar{x}[f(L)]^{k},
$$

and so $L$ and $R$ are both solutions of (4) in the interval $0 \leq x \leq \bar{x}[f(0)]^{k}$. It follows by condition (iv) of Theorem 1 that $L=M=\bar{x}$ and the proof is complete.

\section{REMARKS AND APPLICATIONS}

Remark 1. When $k=1$, condition (iv) of Theorem 1 is a direct consequence of conditions (i)-(iii). To see this, assume for the sake of contradiction, that (4) has a solution $x^{\prime} \neq \bar{x}$. We will assume that $x^{\prime}<\bar{x}$. The case where $x^{\prime}>\bar{x}$ is similar and is omitted. As $x^{\prime}<\bar{x}$, it follows from (3) that $x^{\prime}\left(f\left(x^{\prime}\right)<\bar{x}\right.$. Hence $\bar{x} f\left(x^{\prime}\right)<\bar{x}^{2} / x^{\prime}$, and by using the decreasing nature of $f$, we obtain

$$
f\left(\bar{x} f\left(x^{\prime}\right)\right) \geq f\left(\bar{x}^{2} / x^{\prime}\right) .
$$


Note that $\bar{x}^{2} / x^{\prime}>\bar{x}$, and so by (3),

$$
f\left(\frac{\bar{x}^{2}}{x^{\prime}}\right)>\frac{\bar{x}}{\bar{x}^{2} / x^{\prime}}=\frac{x^{\prime}}{\bar{x}} .
$$

From the above and the assumption that $x^{\prime}$ is a solution of (4) we find

$$
x^{\prime}=\bar{x} f\left(\bar{x}\left(f\left(x^{\prime}\right)\right)>\bar{x} \cdot \frac{x^{\prime}}{\bar{x}}=x^{\prime},\right.
$$

and this contradiction establishes our claim.

A direct consequence of Theorem 1 and the above remark is the following global attractivity result that was established in [4].

Corollary 1. Consider the delay logistic equation

$$
x_{n+1}=\frac{\alpha x_{n}}{1+\beta x_{n-1}}, \quad n=0,1,2, \ldots,
$$

where $\alpha \in(1, \infty)$ and $\beta \in(0, \infty)$. Let $\left\{x_{n}\right\}$ be a solution of $(19)$ with $x_{-1} \geq 0$ and $x_{0}>0$. Then $\lim _{n \rightarrow \infty} x_{n}=(\alpha-1) / \beta$.

The next result is another application of Theorem 1 to the more general delay logistic model

$$
x_{n+1}=\frac{\alpha x_{n}}{1+\beta x_{n-k}}, \quad n=0,1,2, \ldots
$$

where

$$
\alpha \in(1, \infty), \beta \in(0, \infty) \text {, and } k \in \mathbb{N} \text {. }
$$

Corollary 2. In addition to (21) assume that

$$
k \leq \alpha /(\alpha-1) \text {. }
$$

Let $\left\{x_{n}\right\}$ be a solution of (1) with

$$
x_{-k}, \ldots, x_{-1} \in[0, \infty) \text { and } x_{0} \in(0, \infty) .
$$

Then $\lim _{n \rightarrow \infty} x_{n}=(\alpha-1) / \beta$.

Proof. Here $f(x)=\alpha /(1+\beta x)$ and conditions (i)-(iii) of Theorem 1 are clearly satisfied and $\bar{x}=(\alpha-1) / \beta$. Finally, we will prove that if (22) holds the condition (iv) of Theorem 1 is also satisfied. To this end, consider the function

$$
F(x)=x-\bar{x}\left[f\left(\bar{x}(f(x))^{k}\right)\right]^{k}=x-\frac{\alpha-1}{\beta} \cdot \alpha^{k}\left[1+\frac{(\alpha-1) \alpha^{k}}{(1+\beta x)^{k}}\right]^{-k}
$$

for $x \geq 0$. Since $F(0)<0$ and $F(\infty)=\infty$, condition (iv) will be satisfied if we prove that

$$
F^{\prime}(x)>0 \text { for } x \geq 0 \text { and } x \neq \bar{x} \text {. }
$$

We have

$$
F^{\prime}(x)=1-(\alpha-1)^{2} k^{2} \alpha^{2 k}\left[1+\beta x+\frac{(\alpha-1) \alpha^{k}}{(1+\beta x)^{k-1}}\right]^{-k-1},
$$

and so, clearly, $F^{\prime}(x)>0$ for $k=1$. For $k \geq 2$, the function

$$
G(t)=t+(\alpha-1) \alpha^{k} t^{-k+1}
$$


has a minimum in $(0, \infty)$ at $t=t_{0}=\alpha[(k-1)(\alpha-1)]^{1 / k}$. Hence

$$
G(t) \geq G\left(t_{0}\right)=\alpha k[(k-1)(\alpha-1)]^{1 / k} /(k-1),
$$

and so for $k \geq 2$,

$$
F^{\prime}(x) \geq 1-[\alpha(k-1)(\alpha-1)]^{1 / k}\left(\frac{k}{k-1}\right)^{k-1} .
$$

From (22) we see that $\alpha \leq 1+1 /(k-1)$, and so (25) implies that (24) is true. The proof is complete.

Next, we consider the delay difference equation

$$
x_{n+1}=x_{n} e^{r\left(1-x_{n-k}\right)} \quad \text { where } r \in(0, \infty) \text { and } k \in \mathbb{N} .
$$

Here $f(x)=e^{r(1-x)}$ satisfies the hypotheses (i) and (ii) of Theorem 1 but not hypothesis (iii). However, hypothesis $\left(\right.$ iii $^{\prime}$ ) is satisfied provided that

$$
0<r \leq 1 /(k+1) \text {. }
$$

Therefore, the following result is true.

Corollary 3. Assume that (27) holds. Let $\left\{x_{n}\right\}$ be a solution (26) with initial values satisfying (23). Then $\lim _{n \rightarrow \infty} x_{n}=1$.

\section{REFERENCES}

1. M. O. Bergh and W. M. Getz, Stability of discrete age-structured and aggregated delaydifference population models, J. Math. Biology 26 (1988), 551-581.

2. G. Karakostas, Ch. G. Philos, and Y. G. Sficas, The dynamics of some discrete population equations (to appear).

3. V. Lj. Kocic and G. Ladas, Global attractivity in second-order nonlinear difference equation (to appear).

4. S. A. Kuruklis and G. Ladas, Oscillations and global attractivity in a discrete delay logistic model Quart. Appl. Math. (to appear).

5. S. Levin and R. May, A note on difference-delay equations, Theor. Popul. Biol. 9 (1976), 178-187.

6. E. C. Pielou, An introduction to mathematical ecology, Wiley Interscience, New York, 1969.

7. __ Population and community ecology, Gorden and Breach, New York, 1974.

8. A. R. Watkinson, Density-dependence in single-species populations of plants, J. Theor. Biol. 83 (1980), 345-357.

Department of Mathematics, The University of Rhode Island, Kingston, Rhode IsLAND 02881-0816 